\title{
Daffodil Days
}

We'll dance with the daffodils

Touch palms to petals and get the chills

Shake and jive in the breeze

Then run away from the buzzing bees

We can play, we can sing all day

Swap our secrets with a blue jay

We can run in circles or in squares

Our messed up game of musical chairs

We can roll down hills, climb up trees

We can do anything we please

And when the sun starts to set

We'll remember the day we won't forget

Michael Voulgarellis 